\title{
The Effects of Colour in Work Environment: A systematic review
}

\author{
Nattha Savavibool ${ }^{1 *}$, Birgitta Gatersleben ${ }^{2}$, Chumporn Moorapun ${ }^{3}$ \\ $1 \mathrm{PhD}$ Candidate in Multidisciplinary Design Research Program, Faculty of Architecture, \\ King Mongkut's Institute of Technology Ladkrabang, Bangkok, Thailand \\ ${ }^{2}$ School of Psychology, Faculty of Health and Medical Sciences, \\ University of Surrey, Guildford, Surrey, UK \\ 3 Faculty of Architecture, \\ King Mongkut's Institute of Technology Ladkrabang, Bangkok, Thailand \\ nattha@msn.com
}

\begin{abstract}
In the work environment, colour is considered an important design element used to improve aesthetic qualities of the environment and for a company's branding. This paper reviews existing research on the effects of workplace colour on worker's mood, wellbeing and other work-related outcomes. In total 40 papers fitted the inclusion criteria and were included in the review. The results show a significant influence of colour on affect (e.g. mood, emotion), wellbeing (e.g. stress, comfort, wellbeing), and performance (e.g. productivity, performance, creativity). The review concludes with suggestions for further research.
\end{abstract}

Keywords: Colour perception; Colour psychology; Effect of colour; Work environment

eISSN 2398-4295 @ 2018. The Authors. Published for AMER ABRA cE-Bs by e-International Publishing House, Ltd., UK. This is an open-access article under the CC BY-NC-ND license (http://creativecommons.org/licenses/bync-nd/4.0/). Peer-review under responsibility of AMER (Association of Malaysian Environment-Behaviour Researchers), ABRA (Association of Behavioural Researchers on Asians) and cE-Bs (Centre for EnvironmentBehaviour Studies), Faculty of Architecture, Planning \& Surveying, Universiti Teknologi MARA, Malaysia.

DOI: http://dx.doi.org/10.21834/ajbes.v3i13.152 


\subsection{Introduction}

Colour is one of the elements that play a significant role in interior design, especially in the work environment. In the field of environmental psychology, colour is discussed as another environmental factor that has a great impact on human perception and behaviour. People feel stimulated by a warm colour, and a cool colour is calming. Workplace colour can affect the physiological response such as heart rate as well as anxiety and human comfort (Küller et al., 2009). Moreover, colour has an impact on work outcomes such as performance, productivity and creativity (Kwallek et al., 2007, Mehta \& Zhu, 2009). It is often assumed that providing the right colour can have positive effects in the workplace. Moreover, individual colour preference is associated with the emotional response to the environment as well as behaviour in that environment. Therefore, understanding how colour can affect human perceptions and behaviour is essential for creating an efficient work environment.

\subsection{Literature Review}

The aim of this paper is to review the existing studies that examine the effects of colour in the work environment on human perceptions and behaviour, particularly regarding mood and wellbeing and work-related outcomes.

\subsection{Methodology}

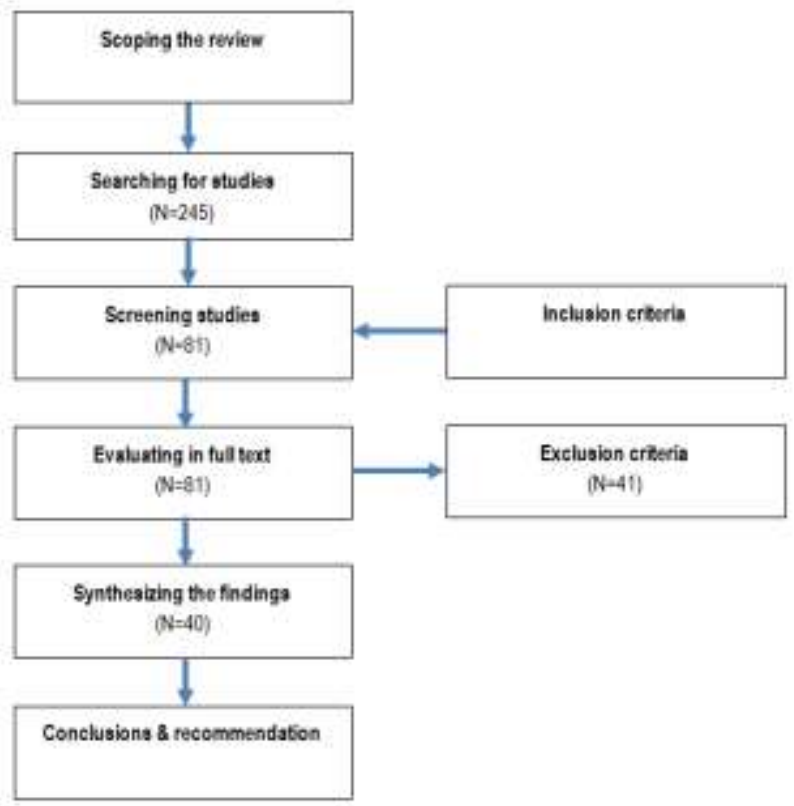

Figure 1: Flow chart of systematic literature review process. 
The procedure of the review included the following steps, as illustrated in Figure.1 • Scoping the review: In the field of psychology, social science, architecture

- Searching: The search was carried out in Scopus and Google Scholar databases.

- Screening process: Following the inclusion criteria, papers were limited to peerreviewed academic literature in English language, published between 1990 and 2016.

- Evaluating the full text of selected papers: The following exclusion criteria were used to exclude articles, studies with no particular colour intervention and no outcomes, studies without a focus on colour and workplace design, descriptive literature, studies without a measurement instrument.

- Analysis and synthesis of the findings

- Conclusion

\section{Results, Discussion, and Recommendation}

Initially, 245 papers were retrieved from the databases. Among them, 81 papers were fit the inclusion criteria for this study. Subsequently, another 41 papers were excluded because they were not empirical studies, and 40 papers were selected for the final review. All studies are summarised in Table 1.

Most of the studies were conducted in the western regions. The cross-cultural studies were mostly conducted across Europe and Asia. Three aspects of colour were studied: hue, saturation and brightness. The majority of the studies focused on studying warm versus cool colours. Warm colours were usually red, orange, yellow, and cool colours were most often blue and green.

The evidence from 40 studies identified that colour of work environment has significant effects on the human in three categories: mood and emotion, physiology and wellbeing, and work-related

outcomes.

Table 1: Categories of study design and the reported effects of colour in the work environment

\begin{tabular}{|c|c|c|c|c|c|c|c|c|c|}
\hline & & & & & & Asse & ssme & ents & \\
\hline & Authors & Study Design & Methods & Participants & Colour sample & 읭 & & & \\
\hline 1 & $\begin{array}{l}\text { Abbas et al. } \\
(2006)\end{array}$ & Experimental & $\begin{array}{l}\text { Laboratory, } \\
\text { ECG }\end{array}$ & $\begin{array}{l}15 \text { students; } \\
\text { Australia }\end{array}$ & $\begin{array}{l}\text { Red, blue, } \\
\text { green }\end{array}$ & $x$ & & & \\
\hline 2 & $\begin{array}{l}\text { Al-Rasheed } \\
\text { (2015) }\end{array}$ & $\begin{array}{l}\text { Experimental } \\
\text { (Cross- } \\
\text { Cultural) }\end{array}$ & $\begin{array}{l}\text { Experimental } \\
\text { room }\end{array}$ & $\begin{array}{l}109 \text { Students; } \\
\text { England, } \\
\text { Saudi Arabia }\end{array}$ & $\begin{array}{l}\text { Red, pink, } \\
\text { Yellow-red, } \\
\text { red-pink, } \\
\text { green, blue- } \\
\text { green, } \\
\text { green-yellow } \\
\end{array}$ & & & $x$ & \\
\hline 3 & \begin{tabular}{|l} 
Bakker et al. \\
$(2013)$
\end{tabular} & Experimental & $\begin{array}{l}\text { Real meeting } \\
\text { rooms, } \\
\text { questionnaires }\end{array}$ & $\begin{array}{l}52 \text { employees; } \\
\text { The } \\
\text { Netherlands }\end{array}$ & Red, blue & $x$ & $x$ & & \\
\hline 4 & \begin{tabular}{|l|} 
Baniani \& \\
Yamamoto (2015)
\end{tabular} & $\begin{array}{l}\text { Experimental } \\
\text { (Cross- }\end{array}$ & $\begin{array}{l}\text { Drawing, } \\
\text { Painting, }\end{array}$ & $\begin{array}{l}319 \text { students; } \\
\text { Iran, Japan, }\end{array}$ & $\begin{array}{l}\text { Colour } \\
\text { schemes }\end{array}$ & & & 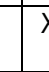 & \\
\hline
\end{tabular}


Savavibool, N., et.al. / Asian Journal of Behavioural Studies (AjBeS), 3(13) Sep / Oct 2018 (p.149-160)

\begin{tabular}{|c|c|c|c|c|c|c|c|c|c|}
\hline & & Cultural) & $\begin{array}{l}\text { Questionnaire } \\
\mathrm{s}\end{array}$ & Foreign & & & & & \\
\hline 5 & $\begin{array}{l}\text { Ceylan et al. } \\
(2008)\end{array}$ & Experimental & $\begin{array}{l}25 \\
\text { photographs }\end{array}$ & $\begin{array}{l}60 \text { managers, } \\
\text { Turkey }\end{array}$ & $\begin{array}{l}\text { Yellow, } \\
\text { orange, pink, } \\
\text { green, red, } \\
\text { red violet, } \\
\text { blue, blue } \\
\text { violet }\end{array}$ & & & $X$ & \\
\hline 6 & Dijkstra (2008) & Experimental & $\begin{array}{l}\text { Laboratory, } \\
\text { Stress Arousal } \\
\text { Checklist }\end{array}$ & $\begin{array}{l}89 \text { students; } \\
\text { Turkey }\end{array}$ & $\begin{array}{l}\text { Green, } \\
\text { Orange, white }\end{array}$ & & $\bar{X}$ & & \\
\hline 7 & Dul et al. (2011) & Survey & $\begin{array}{l}\text { Creative } \\
\text { Personality } \\
\text { Scale }\end{array}$ & $\begin{array}{l}274 \text { workers; } \\
\text { The } \\
\text { Netherlands }\end{array}$ & $\begin{array}{l}\text { Warm colours, } \\
\text { cool colours }\end{array}$ & & & $\mathrm{X}$ & \\
\hline 8 & \begin{tabular}{|l} 
Elliot et al. \\
(2007)
\end{tabular} & $\begin{array}{l}\text { Experimental } \\
\text { (Cross- } \\
\text { Cultural) }\end{array}$ & $\begin{array}{l}\text { Laboratory \& } \\
\text { classroom } \\
\text { IQ test, task }\end{array}$ & $\begin{array}{l}282 \text { students; } \\
\text { USA, Germany }\end{array}$ & $\begin{array}{l}\text { Red, green } \\
\text { black, white, } \\
\text { gray }\end{array}$ & $X$ & & $X$ & \\
\hline 9 & Franz (2006) & Experimental & $\begin{array}{l}\text { Web } \\
\text { experiment }\end{array}$ & $\begin{array}{l}125 \\
\text { participants; } \\
\text { Germany }\end{array}$ & $\begin{array}{l}16 \text { hue, } \\
4 \text { saturation } \\
\& \text { brightness } \\
\text { levels. }\end{array}$ & $\mathrm{X}$ & & & \\
\hline 10 & $\begin{array}{l}\text { Gao et al. } \\
(2007)\end{array}$ & $\begin{array}{l}\text { Experimental } \\
\text { (Cross- } \\
\text { Cultural) }\end{array}$ & $\begin{array}{l}\text { Experimental } \\
\text { room }\end{array}$ & $\begin{array}{l}440 \text { students; } \\
\text { Hong Kong, } \\
\text { Japan, } \\
\text { Thailand, } \\
\text { Taiwan, Italy, } \\
\text { Spain, Sweden }\end{array}$ & 214 colours & $X$ & & & \\
\hline 11 & $\begin{array}{l}\text { Gyu \& Park } \\
\text { (2013) }\end{array}$ & $\begin{array}{l}\text { Experimental } \\
\text { (Cross- } \\
\text { Cultural) } \\
\end{array}$ & Model & $\begin{array}{l}92 \text { participants, } \\
\text { USA, Korea }\end{array}$ & $\begin{array}{l}\text { Red, yellow, } \\
\text { green, blue, } \\
\text { purple, white } \\
\end{array}$ & & & & $\mathrm{X}$ \\
\hline 12 & $\begin{array}{l}\text { Hidayetoglu et } \\
\text { al. (2012) }\end{array}$ & Experimental & $\begin{array}{l}9 \text { pictures, } \\
9 \text { videos }\end{array}$ & $\begin{array}{l}102 \text { students; } \\
\text { Turkey }\end{array}$ & $\begin{array}{l}\text { Red, blue } \\
\text { grey, with low } \\
\& \text { high } \\
\text { brightness }\end{array}$ & $X$ & & & \\
\hline 13 & $\begin{array}{l}\text { Kamaruzzaman } \\
\text { et al. }(2010)\end{array}$ & Survey & $\begin{array}{l}\text { Questionnaire } \\
\text { s, interviews }\end{array}$ & $\begin{array}{l}105 \text { employees; } \\
\text { Malaysia }\end{array}$ & $\begin{array}{l}\text { Colour } \\
\text { schemes }\end{array}$ & $X$ & & $X$ & $\mathrm{X}$ \\
\hline 14 & $\begin{array}{l}\text { Kaya et al. } \\
(2004)\end{array}$ & Experimental & $\begin{array}{l}\text { Experimental } \\
\text { room }\end{array}$ & $\begin{array}{l}98 \text { students; } \\
\text { USA }\end{array}$ & $\begin{array}{l}\text { Red, yellow, } \\
\text { green, blue, } \\
\text { purple, white, } \\
\text { gray, black } \\
5 \\
\text { intermediate } \\
\text { hues: }\end{array}$ & $X$ & & & \\
\hline 15 & $\begin{array}{l}\begin{array}{l}\text { Kurt \& Osueke } \\
(2014)\end{array} \\
\end{array}$ & Survey & $\begin{array}{l}\text { Questionnaire } \\
\text { s, interviews }\end{array}$ & $\begin{array}{l}490 \text { students, } \\
\text { staffs; Cyprus }\end{array}$ & $\begin{array}{l}\text { Colour } \\
\text { schemes }\end{array}$ & $X$ & & & \\
\hline 16 & \begin{tabular}{|l|} 
Kwallek \& \\
Lewis (1990)
\end{tabular} & Experimental & $\begin{array}{l}\text { Experimental } \\
\text { room, tests, } \\
\text { questionnaires }\end{array}$ & $\begin{array}{l}675 \text { students; } \\
\text { USA }\end{array}$ & $\begin{array}{l}\text { Red, green, } \\
\text { white }\end{array}$ & $x$ & & $X$ & $\mathrm{X}$ \\
\hline 17 & $\begin{array}{l}\text { Kwallek et al. } \\
(1996)\end{array}$ & Experimental & $\begin{array}{l}\text { Experimental } \\
\text { room, tests, } \\
\text { questionnaires }\end{array}$ & $\begin{array}{l}90 \text { workers; } \\
\text { USA }\end{array}$ & $\begin{array}{l}\text { Red, orange, } \\
\text { yellow, blue, } \\
\text { green, purple, } \\
\text { white, gray, } \\
\text { beige }\end{array}$ & $X$ & & $\bar{X}$ & $\mathrm{X}$ \\
\hline
\end{tabular}


Savavibool, N., et.al. / Asian Journal of Behavioural Studies (AjBeS), 3(13) Sep / Oct 2018 (p. 149-160)

\begin{tabular}{|c|c|c|c|c|c|c|c|c|c|}
\hline 18 & $\begin{array}{l}\text { Kwallek et al. } \\
\text { (1997) }\end{array}$ & Experimental & $\begin{array}{l}\text { Experimental } \\
\text { room, tests, } \\
\text { questionnaires }\end{array}$ & $\begin{array}{l}90 \text { workers; } \\
\text { USA }\end{array}$ & $\begin{array}{l}\text { Red, blue- } \\
\text { green, white }\end{array}$ & $x$ & & $X$ & $x$ \\
\hline 19 & $\begin{array}{l}\text { Kwallek et al. } \\
(2007)\end{array}$ & Experimental & $\begin{array}{l}\text { Experimental } \\
\text { room } \\
\text { Questionnaire } \\
\mathrm{s}\end{array}$ & $\begin{array}{l}90 \text { workers; } \\
\text { USA }\end{array}$ & $\begin{array}{l}\text { Red, blue- } \\
\text { green, } \\
\text { white }\end{array}$ & & & $\mathrm{X}$ & \\
\hline 20 & $\begin{array}{l}\text { Küller et al. } \\
(2006)\end{array}$ & $\begin{array}{l}\text { Experimental } \\
\text { (Cross- } \\
\text { Cultural) }\end{array}$ & $\begin{array}{l}\text { Real work } \\
\text { environments, } \\
\text { Questionnaire } \\
\text { s }\end{array}$ & $\begin{array}{l}\text { 998 employees; } \\
\text { Argentina, } \\
\text { Saudi Arabia, } \\
\text { Sweden, UK }\end{array}$ & $\begin{array}{l}\text { Monotonous, } \\
\text { neutral, } \\
\text { colourful }\end{array}$ & $X$ & & & \\
\hline 21 & $\begin{array}{l}\text { Küller et al. } \\
(2009)\end{array}$ & Experimental & $\begin{array}{l}\text { Experimental } \\
\text { room, EEG, } \\
\text { EKG, } \\
\text { interview, } \\
\text { questionnaires }\end{array}$ & $\begin{array}{l}57 \text { students; } \\
\text { Sweden }\end{array}$ & $\begin{array}{l}\text { Red, blue } \\
\text { Colourful, gray }\end{array}$ & 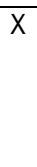 & $\mathrm{X}$ & $\mathrm{X}$ & \\
\hline 22 & Lengen (2015) & Experimental & $\begin{array}{l}\text { Painting, } \\
\text { interview }\end{array}$ & $\begin{array}{l}20 \text { participants; } \\
\text { Switzerland }\end{array}$ & $\begin{array}{l}\text { Blue, green, } \\
\text { grey, white }\end{array}$ & & $X$ & & \\
\hline 23 & $\begin{array}{l}\text { Lebedkova et } \\
\text { al. (2012) }\end{array}$ & Experimental & $\begin{array}{l}\text { Experimental } \\
\text { room, } \\
\text { Questionnaire } \\
\text { s }\end{array}$ & $\begin{array}{l}10 \text { participants, } \\
\text { Russia }\end{array}$ & $\begin{array}{l}\text { Orange, ochre, } \\
\text { blue \& yellow, } \\
\text { green, dark } \\
\text { blue }\end{array}$ & & $\mathrm{X}$ & $\mathrm{X}$ & \\
\hline 24 & Liu et al. (2014) & Experimental & $\begin{array}{l}\text { Questionnaire } \\
\mathrm{s}\end{array}$ & $\begin{array}{l}75 \text { patients; } \\
\text { China }\end{array}$ & 27 colours & $\mathrm{X}$ & & & $\mathrm{X}$ \\
\hline 25 & Manav (2007) & Experimental & $\begin{array}{l}\text { Experimental } \\
\text { room, } \\
\text { questionnaires }\end{array}$ & $\begin{array}{l}20 \text { students, } \\
\text { Turkey }\end{array}$ & $\begin{array}{l}\text { Light blue and } \\
\text { light yellow }\end{array}$ & $\mathrm{X}$ & & & \\
\hline 26 & $\begin{array}{l}\text { McCoy and } \\
\text { Evans (2002) }\end{array}$ & Experimental & photographs & $\begin{array}{l}60 \text { students; } \\
\text { USA }\end{array}$ & $\begin{array}{l}\text { Red, yellow, } \\
\text { red-violet, } \\
\text { green, blue, } \\
\text { blue violet }\end{array}$ & & & $\mathrm{X}$ & \\
\hline 27 & $\begin{array}{l}\text { Mehta \& Zhu } \\
(2009)\end{array}$ & Experimental & Computer & $\begin{array}{l}666 \\
\text { participants; } \\
\text { Canada }\end{array}$ & Red, blue & & & $\mathrm{X}$ & \\
\hline 28 & Ou et al. (2012) & $\begin{array}{l}\text { Experimental } \\
\text { (Cross- } \\
\text { Cultural) }\end{array}$ & $\begin{array}{l}\text { Experimental } \\
\text { room }\end{array}$ & $\begin{array}{l}223 \text { participants; } \\
\text { UK, Taiwan, } \\
\text { France, Iran } \\
\text { Germany, Spain, } \\
\text { Sweden, } \\
\text { Argentina }\end{array}$ & $\begin{array}{l}190 \text { colour } \\
\text { pairs }\end{array}$ & $X$ & & & $X$ \\
\hline 29 & $\begin{array}{l}\text { Öztürk et al. } \\
(2012)\end{array}$ & Experimental & $\begin{array}{l}\text { Experimental } \\
\text { room }\end{array}$ & $\begin{array}{l}60 \text { staffs; } \\
\text { Turkey }\end{array}$ & $\begin{array}{l}\text { Achromatic, } \\
\text { Chromatic }\end{array}$ & $X$ & & $x$ & \\
\hline 30 & $\begin{array}{l}\text { Park \& Guerin } \\
(2002)\end{array}$ & $\begin{array}{l}\text { Experimental } \\
\text { (Cross- } \\
\text { Cultural) }\end{array}$ & $\begin{array}{l}\text { Experimental } \\
\text { room, } \\
\text { questionnaires }\end{array}$ & $\begin{array}{l}425 \text { students; } \\
\text { USA, England, } \\
\text { Korea, Japan }\end{array}$ & $\begin{array}{l}\text { Hue, value, } \\
\text { chroma, }\end{array}$ & & & & $\mathrm{X}$ \\
\hline 31 & Park (2009) & Experimental & Model & $\begin{array}{l}153 \\
\text { participants; } \\
\text { USA }\end{array}$ & $\begin{array}{l}\text { Red, yellow, } \\
\text { green, blue, } \\
\text { purple, white, } \\
9 \text { brightness \& } \\
\text { saturation }\end{array}$ & & & & $X$ \\
\hline 32 & $\begin{array}{l}\text { Poursafar et al. } \\
(2016)\end{array}$ & $\begin{array}{l}\text { Survey } \\
\text { (Cross- }\end{array}$ & $\begin{array}{l}\text { Questionnaire } \\
\mathrm{s}\end{array}$ & $\begin{array}{l}202 \text { architects; } \\
\text { Iran, India }\end{array}$ & $\begin{array}{l}\text { Colour } \\
\text { schemes }\end{array}$ & & & $\mathrm{X}$ & $x$ \\
\hline
\end{tabular}




\begin{tabular}{|c|c|c|c|c|c|c|c|c|c|}
\hline & & Cultural) & & & & & & & \\
\hline 33 & Saito (1996) & $\begin{array}{l}\text { Survey } \\
\text { (Cross- } \\
\text { Cultural) }\end{array}$ & $\begin{array}{l}\text { Colour chart, } \\
\text { questionnaires } \\
\text {. }\end{array}$ & $\begin{array}{l}1600 \\
\text { participants, } \\
\text { Japan, Korea, } \\
\text { Taiwan }\end{array}$ & $\begin{array}{l}62 \\
\text { chromatic } \\
3 \\
\text { achromatic }\end{array}$ & & & & $\mathrm{X}$ \\
\hline 34 & $\begin{array}{l}\text { Sorokowski et } \\
\text { al. (2014) }\end{array}$ & $\begin{array}{l}\text { Survey } \\
\text { (Cross- } \\
\text { Cultural) }\end{array}$ & $\begin{array}{l}\text { Colour chart, } \\
\text { interviews }\end{array}$ & $\begin{array}{l}308 \\
\text { participants; } \\
\text { Papua, Poland }\end{array}$ & $\begin{array}{l}\text { Red, orange, } \\
\text { yellow, green, } \\
\text { blue, purple \& } \\
6 \text { colours in- } \\
\text { between }\end{array}$ & & & & $X$ \\
\hline 35 & $\begin{array}{l}\text { Stone \& English } \\
\text { (1998) }\end{array}$ & Experimental & $\begin{array}{l}\text { Experimental } \\
\text { room, MAACL, } \\
\text { questionnaires }\end{array}$ & $\begin{array}{l}122 \text { students; } \\
\text { USA }\end{array}$ & Red, blue & $\bar{X}$ & & $\bar{X}$ & \\
\hline 36 & Stone (2001) & Experimental & $\begin{array}{l}\text { Experimental } \\
\text { room, MAACL, } \\
\text { questionnaires }\end{array}$ & $\begin{array}{l}144 \text { students; } \\
\text { USA }\end{array}$ & $\begin{array}{l}\text { Red, blue, } \\
\text { white }\end{array}$ & $X$ & & 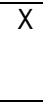 & \\
\hline 37 & Stone (2003) & Experimental & $\begin{array}{l}\text { Experimental } \\
\text { room, MAACL, } \\
\text { questionnaires }\end{array}$ & $\begin{array}{l}128 \text { students; } \\
\text { USA }\end{array}$ & Red, blue & $X$ & & $X$ & \\
\hline 38 & $\begin{array}{l}\text { Taylor, Clifford } \\
\text { and Flanklin } \\
(2013)\end{array}$ & $\begin{array}{l}\text { Experimental } \\
\text { (Cross- } \\
\text { Cultural) }\end{array}$ & Computer & $\begin{array}{l}95 \text { participants; } \\
\text { Namibia, } \\
\text { England }\end{array}$ & $\begin{array}{l}\text { Red, orange, } \\
\text { yellow, green, } \\
\text { cyan, blue, } \\
\text { purple \& } 8 \\
\text { saturation }\end{array}$ & & & & $X$ \\
\hline 39 & $\begin{array}{l}\text { Valdez \& } \\
\text { Mehrabian } \\
(1994)\end{array}$ & Experimental & $\begin{array}{l}\text { Color cards, } \\
\text { PAD scales }\end{array}$ & $\begin{array}{l}396 \text { students; } \\
\text { USA }\end{array}$ & $\begin{array}{l}\text { Red, yellow, } \\
\text { green, blue, } \\
\text { purple, } \\
\text { red-purple, } \\
\text { yellow-red, } \\
\text { green- } \\
\text { yellow, blue- } \\
\text { green, } \\
\text { purple-blue }\end{array}$ & $X$ & & & $\mathrm{X}$ \\
\hline 40 & $\begin{array}{l}\text { Yildirim et al. } \\
(2015)\end{array}$ & Survey & $\begin{array}{l}\text { Questionnaire } \\
\mathrm{s}\end{array}$ & $\begin{array}{l}909 \text { students; } \\
\text { Turkey }\end{array}$ & $\begin{array}{l}\text { Blue, pink, } \\
\text { cream }\end{array}$ & $X$ & & & \\
\hline \multicolumn{6}{|c|}{ Total } & 21 & 6 & 18 & 16 \\
\hline
\end{tabular}

\subsection{Colour preference}

In total 16 studies were included that examined colour preferences. Most of the studies were cross-cultural study. Blue and green are consistently found to be the most favourite colours (Kamaruzzaman et al., 2010; Kwallek et al., 1997; Liu et al., 2014; Park, 2009; Poursafar et al., 2016; Valdez \& Mehrabian, 1994). However, colour preference is not universal (Taylor, Clifford and Flanklin, 2013) and are influenced by differences in age, gender, cultural aspect (Park \& Guerin, 2002; Saito, 1996; Sorokowski et al., 2014), background and experience (Baniani \& Yamamoto, 2015).

In the workplace, the preference of colours can influence on worker's mood, wellbeing 
and performance. (Kamaruzzaman et al., 2010; Poursafar et al., 2016). White is the most favourite neutral colour (Poursafar et al., 2016) and workers prefer to work in a white environment (Kwallek \& Lewis, 1990; Kwallek et al.,1996).

\subsection{Mood and emotion}

In total 21 studies focused on mood and emotion. Most of the studies used a subjective measure of mood such as The Multiple Affect Adjective Check List (MAACL); The PAD (Pleasure, Arousal, and Dominance).

The emotional responses to colour are related to the meaning of colours. Green evokes the most positive emotional responses and is associated with relaxation, and happiness (Kaya et al., 2004). A cross-cultural study found positive emotional status when working in the colourful environment. The good colour scheme will enhance overall mood of worker (Küller et al., 2006). Blue is perceived more positive than red in the open-plan environment (Stone, 2001) but other studies suggest it can also be perceived as depressive (Stone \& English, 1998), and less attractive (Yildirim et al., 2015). The red environment can be perceived as stimulating as well as distracting (Kwallek \& Lewis, 1990; Kwallek et al., 1997). White walls tend to be perceived boring and uninteresting (Kurt \& Osueke, 2014).

\subsection{Physiology and wellbeing}

In total six studies focused on health outcomes using physiological measures. This included Electroencephalogram (EEG) and electrocardiogram (EKG or ECG) to measure brain's activity and heart rate variation.

Some colours have a greater impact on heart rate than others (Abbas et al., 2006). Working in the red or colourful room with visual complexity put the brain into a more exciting state, caused the slowing of the heart rate and overload. Blue was reported to have a drowsy and sleepy effect (Küller et al., 2009). Good workplace colour combinations can have a positive influence on visual working capacity and increase comfort (Lebedkova et al., 2012). Perception and experience in blue and green space are associated with a sense of wellbeing.

\subsection{Work-related outcomes}

In this section, 18 studies regarding the effects of workplace colour on work-related outcomes were divided into the following main topics: performance, productivity and creativity.

\subsubsection{Performance}

In total 11 studies on colour and performance were included which were all conducted in laboratory settings. A colourful workplace tended to enhance performance more than a workplace with an achromatic scheme (Öztürk et al., 2012). Some colour combinations increase a positive influence on visual working performance; speed of work and accuracy (Lebedkova et al., 2012). Working in the white environment resulted in the most errors being made (Kwallek \& Lewis, 1990; Kwallek et al., 1996). Red was reported to have both negative and positive effect (Elliot et al., 2007; Kwallek et al., 1996; Küller et al., 2009).

The level of performance depended on the type of task and task demand (Stone \& English, 1998, Stone, 2001, Stone, 2003). Red enhances cognitive task performance 
whereas working in the blue enhances creative task performance (Mehta \& Zhu, 2009).

\subsubsection{Productivity}

Four studies reported the effect of workplace colour on human's productivity. Neutral colours, cool blue colours (Kamaruzzaman et al., 2010) as well as a balance between warm and cool colours can enhance productivity. By contrast, cool colours in the context of architectural office were found to reduce productivity (Poursafar et al., 2016). Interestingly, one study conducted in a real life work situation reported no effect of colour on perceived productivity (Bakker et al., 2013).

\subsubsection{Creativity}

Four studies showed that workplace colour had the influence on creativity. Cool colours have a significant negative effect on creativity potential (McCoy and Evans, 2002). In contrast, Ceylan et al. (2008) reported that the cool workplace colours enhanced creativity, supporting the managers to think and generate ideas. Positive effects may be because cool colours are calming whereas warm colours are overstimulating making it difficult for people to concentrate.

\subsection{Discussions}

It is very clear that workplace colour has the significant effect on mood, physiology and wellbeing and work-related outcomes. Both consistency and contradictory results were found. The individual difference regarding culture, gender, age and background may influence the perception of workplace colour and colour preferences. Studies with students in hypothetical laboratory studies may not be comparable to experiences in the actual work environment. Moreover, the existing research almost focuses on a limited number of colours, primarily red, blue and green. There is very little research which examines colour combinations which may be more representative for workplace colour in a real everyday work environment.

\subsection{Conclusion}

Strong evidence was established that colour in work environment plays a significant role in human's perception and behaviour especially mood, wellbeing and performance. The use of colour in the workplace can enhance positive mood, contribute the sense of wellbeing and lead to a positive outcome. Understanding the maximum dimension of how difference workplace colours influence humans is essential. More research is needed to examine the following factors: the combination of colour, the context of the work environment, crosscultural factors and individual differences.

\section{Acknowledgement}

The author would like to thank Dr Birgitta Gatersleben and Ass. Prof. Dr Chumporn Moorapun 
for their advice and general support. This study has been funded by Sripatum University under the Faculty Development Grant Program.

\section{References}

Abbas, N., Kumar, D., \& Mclachlan, N. (2006, January). The psychological and physiological effects of light and colour on space users. In Engineering in Medicine and Biology Society, 2005. IEEE-EMBS 2005. 27th Annual International Conference of the (pp. 1228-1231). IEEE.

Al-Rasheed, A. S. (2015). An experimental study of gender and cultural differences in hue preference. Frontiers in psychology, 6 .

Bakker, I., van der Voordt, T. J., de Boon, J., \& Vink, P. (2013). Red or blue meeting rooms: does it matter? The impact of colour on perceived productivity, social cohesion and wellbeing. Facilities, 31(1/2), 68-83.

Baniani, M., \& Yamamoto, S. (2015). A comparative study on correlation between personal background and interior color preference. Color Research \& Application, 40(4), 416-424.

Ceylan, C., Dul, J., \& Aytac, S. (2008). Can the office environment stimulate a manager's creativity? Human Factors and Ergonomics in Manufacturing \& Service Industries, 18(6), 589-602.

Dijkstra, K., Pieterse, M. E., \& Pruyn, A. T. H. (2008). Individual differences in reactions towards color in simulated healthcare environments: The role of stimulus screening ability. Journal of Environmental Psychology, 28(3), 268-277.

Dul, J., Ceylan, C., \& Jaspers, F. (2011). Knowledge workers' creativity and the role of the physical work environment. Human resource management, 50(6), 715-734.

Elliot, A. J., Maier, M. A., Moller, A. C., Friedman, R., \& Meinhardt, J. (2007). Color and psychological functioning: the effect of red on performance attainment. Journal of experimental psychology: General, 136(1), 154.

Franz, G. (2006, September). Space, color, and perceived qualities of indoor environments. In Environment, health and sustainable development Proceedings of the 19th International Association for People-Environment Studies Conference (IAPS 2006). Seattle, WA, USA Hogrefe \& Huber.

Gao, X. P., Xin, J. H., Sato, T., Hansuebsai, A., Scalzo, M., Kajiwara, K. \& Billger, M. (2007). Analysis of cross-cultural color emotion. Color Research \& Application, 32(3), 223-229. 
Gyu, J., \& Park, C. (2013). Color perception in pediatric patient room design: American versus Korean pediatric patients. HERD: Health Environments Research \& Design Journal, 6(4), 10-26.

Hidayetoglu, M. L., Yildirim, K., \& Akalin, A. (2012). The effects of color and light on indoor wayfinding and the evaluation of the perceived environment. Journal of environmental psychology, 32(1), 50-58.

Kamaruzzaman, S. N., \& Zawawi, E. M. A. (2010). Influence of Employees' Perceptions of Colour Preferences on Productivity in Malaysian Office Buildings. Journal of Sustainable Development, 3(3), 283.

Kaya, N., \& Epps, H. H. (2004). Relationship between color and emotion: A study of college students. College student journal, 38(3), 396.

Kurt, S., \& Osueke, K. K. (2014). The effects of color on the moods of college students. SAGE Open, 4(1), 2158244014525423.

Kwallek, N., \& Lewis, C. M. (1990). Effects of environmental colour on males and females: A red or white or green office. Applied Ergonomics, 21(4), 275-278.

Kwallek, N., Lewis, C. M., Lin-Hsiao, J. W. D., \& Woodson, H. (1996). Effects of nine monochromatic office interior colors on clerical tasks and worker mood. Color Research and Application, 21(6), 448-458.

Kwallek, N., Woodson, H., Lewis, C. M., \& Sales, C. (1997). Impact of three interior color schemes on worker mood and performance relative to individual environmental sensitivity. Color Research and Application, 22(2), 121-132.

Kwallek, N., Soon, K., \& Lewis, C. M. (2007). Work week productivity, visual complexity, and individual environmental sensitivity in three offices of different color interiors. Color Research \& Application, 32(2), 130-143.

Küller, R., Ballal, S., Laike, T., Mikellides, B., \& Tonello, G. (2006). The impact of light and colour on psychological mood: a cross-cultural study of indoor work environments. Ergonomics, 49(14), 1496-1507.

Küller, R., Mikellides, B., \& Janssens, J. (2009). Color, arousal, and performance-A comparison of three experiments. Color Research \& Application, 34(2), 141-152.

Lengen, C. (2015). The effects of colours, shapes and boundaries of landscapes on perception, emotion and mentalising processes promoting health and well-being. Health \& place, 35, 166-177. 
Lebedkova, S. M., Panteleeva, N. S., \& Stepanova, I. V. (2012). Influence on Visual Working Capacity of Colour Decoration of the Workplace for Work with a Computer. Light and Engineering, 20(3), 40.

Liu, W., Ji, J., Chen, H., \& Ye, C. (2014). Optimal Color Design of Psychological Counseling Room by Design of Experiments and Response Surface Methodology. PloS one, 9(3), e90646.

Manav, B. (2007). An experimental study on the appraisal of the visual environment at offices in relation to colour temperature and illuminance. Building and environment, 42(2), 979-983.

McCoy, J. M., \& Evans, G. W. (2002). The potential role of the physical environment in fostering creativity. Creativity Research Journal, 14(3-4), 409-426.

Mehta, R., \& Zhu, R. J. (2009). Blue or red? Exploring the effect of color on cognitive task performances. Science, 323(5918), 1226-1229.

Nasar, J. (1994). Urban design aesthetics: The evaluative qualities of building exteriors. Environment and Behaviour, 26 (3), 377-401.

Ou, L. C., Ronnier Luo, M., Sun, P. L., Hu, N. C., Chen, H. S., Guan, S. S., ... \& Billger, M. (2012). A cross-cultural comparison of colour emotion for two-colour combinations. Color Research \& Application, 37(1), 23-43.

Öztürk, E., Yılmazer, S., \& Ural, S. E. (2012). The effects of achromatic and chromatic color schemes on participants' task performance in and appraisals of an office environment. Color Research \& Application, 37(5), 359-366.

Park, Y., \& Guerin, D. A. (2002). Meaning and preference of interior color palettes among four cultures. Journal of interior design, 28(1), 27-39.

Park, J. G. (2009). Color perception in pediatric patient room design: Healthy children vs. pediatric patients. HERD: Health Environments Research \& Design Journal, 2(3), 6-28.

Poursafar, Z., Devi, N. R., \& Rodrigues, L. R. (2016). Evaluation of color and lighting Preferences in architects' offices for enhancing productivity. International Journal of Current Research and Review, 8(3), 1.

Saito, M. (1996). Comparative studies on color preference in Japan and other Asian regions, with special emphasis on the preference for white. Color Research \& Application, 21(1), 3549. 
Sorokowski, P., Sorokowska, A., \& Witzel, C. (2014). Sex differences in color preferences transcend extreme differences in culture and ecology. Psychonomic bulletin \& review, 21(5), 1195-1201.

Stone, N. J., \& English, A. J. (1998). Task type, posters, and workspace color on mood, satisfaction, and performance. Journal of Environmental Psychology, 18(2), 175-185.

Stone, N. J. (2001). Designing effective study environments. Journal of Environmental Psychology, 21(2), 179-190.

Stone, N. J. (2003). Environmental view and color for a simulated telemarketing task. Journal of Environmental Psychology, 23(1), 63-78.

Taylor, C., Clifford, A., \& Franklin, A. (2013). Color preferences are not universal. Journal of Experimental Psychology: General, 142(4), 1015.

Valdez, P., \& Mehrabian, A. (1994). Effects of color on emotions. Journal of experimental psychology: General, 123(4), 394.

Yildirim, K., Cagatay, K., \& Ayalp, N. (2015). Effect of wall colour on the perception of classrooms. Indoor and Built Environment, 24(5), 607-616. 